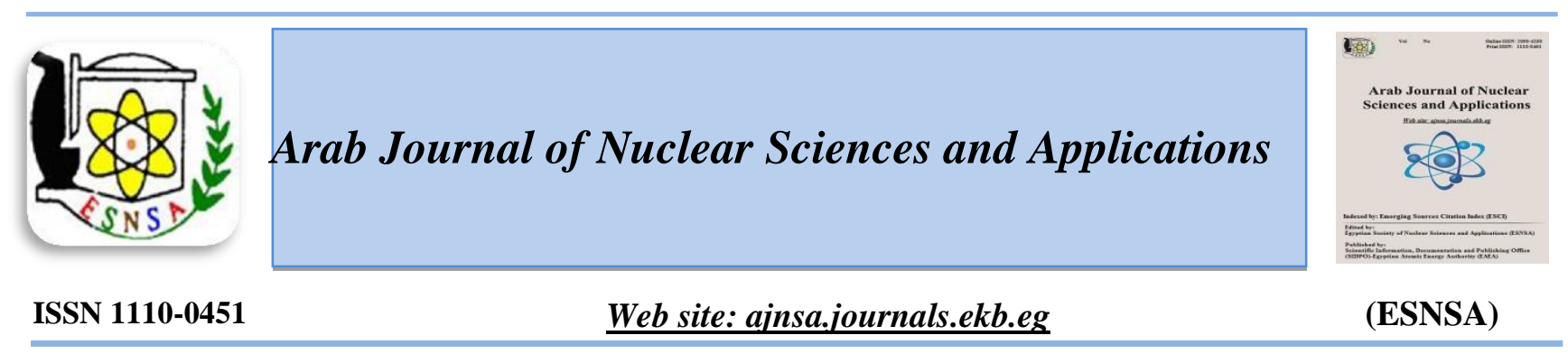

\title{
A Comparative Study and Dose Evaluation of Photon Beam for water phantom, 2D-array and Treatment Planning System in Small Field Sizes
}

\author{
Sherihan Hassn ${ }^{1}$, Nashaat A. Deiab ${ }^{2}$ and Arafa H. Aly ${ }^{3}$ \\ ${ }^{I}$ Minia Oncology Center, Ministry of Health and Population, Minia, Egypt \\ ${ }^{2}$ National Cancer Institute (NCI), Cairo University, Cairo, Egypt \\ ${ }^{3}$ TH-PPM Group, Physics Department, Faculty of Sciences, Beni-Suef University, Egypt
}

Received $22^{\text {th }}$ May 2019 This study assessed the clinical usefulness of the 2D-Array Seven29 by comparing the dose of Photon Accepted $25^{\text {th }}$ Sep. 2019 Beam for water phantom, 2D-array and Treatment Planning System in five Field sizes. The experimental equipment included the linear accelerator, treatment planning system (TPS), water phantom, 2D-Array, solid phantoms, and two types of ionization chambers (Semiflex and Pinpoint). The distance between the ray source and the center of the ion chamber was fixed to SSD of $100 \mathrm{~cm}$ during the experiment. The field size was $10 \times 10 \mathrm{~cm}^{2}$ and the radiation energies of the photon beam were $6 \mathrm{MV}$ and $15 \mathrm{MV}$. The energy fields sizes involved $(1 \times 1,2 \times 2,3 \times 3,4 \times 4,5 \times 5$,). The relative deviation ratio of the TPS in water phantom and TPS to 2 darray in the solid phantom was calculated. The measured doses in standard phantom were lower than TPS values for small field dimensions (less than $3 \times 3 \mathrm{~cm}^{2}$ ). The data measured by 2D-Array for fields $2 \times 2$ and $3 \times 3 \mathrm{~cm}^{2}$ within $\pm 2 \%$ indicated a good accuracy of measurement planning system for field size $1 \times 1 \mathrm{~cm}^{2}$. The measured doses in the standard phantom was lower than the TPS values for small field dimensions and the data measured by $2 \mathrm{D}$-Array for 6 MV shows mild differences between the TPS and the experimental data. A slight difference appeared between the TPS and the experimental data using 2D-Array for $15 \mathrm{MV}$. The results were the same for the TPS and the measured data, at only a field dimension of $1 \times 1 \mathrm{~cm}^{2}$. The measured data increased as field area enlarged from $2 \times 2$ to $5 \times 5 \mathrm{~cm}^{2}$ by a constant value of $4 \%$.

Keywords: 2D-Array seven29, Photon beam dose, Small field sizes, Treatment planning system

\section{Introduction}

Radiotherapy is primarily intended to provide a dose that is appropriately absorbed to a specific target size with a peripheral secretion dose of the total tissue. The uncertainty within the dose given to the patient must be between $3-5 \%$ (1 variance standard deviation, SD)[1]. Although, it has been found in some cases, for example, in palliative treatment, the unit of a higher uncertainty area is acceptable [2]. Lutz et al. (2011) [2] have conducted an experiment to reach a precise calculation of the small field-size dose by assessing the accuracy of the calculation of the planning system. Two steps were carried out to estimate the measured dose using pinpoint ionization chambers calculated by the TPS based on the latest IAEATRS-398. Researchers concluded that the average dimensions of the small fields were $7 \%$ for $6 \mathrm{MV}$ and $3 \%$ for $15 \mathrm{MV}$. A variation in the results within $\pm 2 \%$ of the TPS calculation for 2D- ARRAY measurements has been shown.

In modern radiotherapy techniques, it is necessary to use a high dose of irradiation fraction. The contents of water in human tissues are very important in this process because they represent $90 \%$ of their components and because of their interaction with radiation. In small fields, the procedure is to adjust activities in the laboratory to international standards in order to re-generate the

Corresponding author: shhassan148@yahoo.com

DOI: 10.21608/ajnsa.2019.13053.1217

(C) Scientific Information, Documentation and Publishing Office (SIDPO)-EAEA 
measurement signal to absorb the dose in water. The goal of irradiation is to confirm that the uncertainty in the patient is 5\% [3].

Taking into account all sources of uncertainty, this indicates that the dose in the purpose of the activity of linear accelerator should not exceed $2 \%$. The small field is relative expression, but it refers to any field smaller than or equal to $4 \times 4 \mathrm{~cm}$ [2]. For small fields, the radiation physics measurements delivered is different from the large fields, and many attempts to measure that dose failed because of the limited size for an ion chamber, since the measured dose is an intermediate dose of the total size of the chamber, the results of the ion chamber lose its spatial accuracy once the force field is smaller than the chamber. Therefore, the results of the ion chamber are less than the acceptable of the dose in the middle of the dim field and are more accurate in the dimension of the penumbra at the edge of the small field [4]. The results of Ji et al. (2012) [4] were obtained with the field volume $3 \times 3 \mathrm{~cm}^{2}$ taking into account the size of the standard treatment field in both dose measurements and in dose calculations. Treatment planning system commissioning requires the input of beam data, especially the processing units that need obtaining the characteristics of depth dose, beam profiles, and RDFs [5]. There are three balancing factors confirming the sphere size as a small field thought to be a small field or not: (1) the dimensions of the beam are equivalent to what is expected on the detectors. (2) appropriate dimension of the detector used in the measurements. (3) Small scattered radiation due to small field dimensions [6].

The difficulty of reaching the correct field dose measurements is similar to the factors affecting certain measurements within large field measurements such as acute dose gradients in penumbra regions as well as the loss of equilibrium of charged particles within the construction area, yet they are distinguished [7]. The loss of the charged side particle balance usually results in a decreased dose in the central axis, rather than simply at the edges of the beam and inside the construction areas. In addition, the narrowing of the beam leads to many lateral side profiles, increasing the need for a higher abstraction solution, not only in the penumbra areas, but also in the central axis [8].

The aim of this study is to reach a precise calculation of the dose of the small field dimensions and to perform this by evaluating the accuracy of the calculation of the planning system, which will be compared with the real measurement of the dose for the same dimensions of the small field using different detectors.

There is a great need for many scientific studies to link the criteria that determine the state of the small field by supporting the beam energy and thus the medium density.

\section{Materials and methods}

In this study, Oncor model of energies $6 \mathrm{MV}$ and $15 \mathrm{MV}$ was used as a linear accelerator. XIO CMS set up was used as treatment planning system (TPS). In order to measure the high-energy Semiflex chamber $(0.125 \mathrm{~cm} 3)$, ionization chambers were used, and the Pinpoint chamber $(0.015 \mathrm{~cm} 3)$ was also used for measuring of fields of the 2D-Array Seven29 model (PTW, Freiburg, Germany) and small inner diameter 3 metric linear unit, which is 2D detector array with 729 ionization chambers organized during $27 \times 27$ matrix with a vigorous space of $27 \times 27 \mathrm{~cm} 2$.

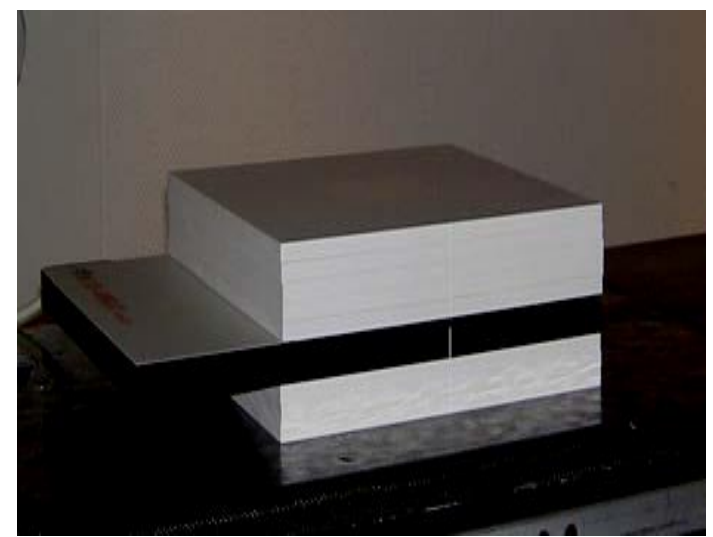

Fig. (1): Two-dimensional array seven29

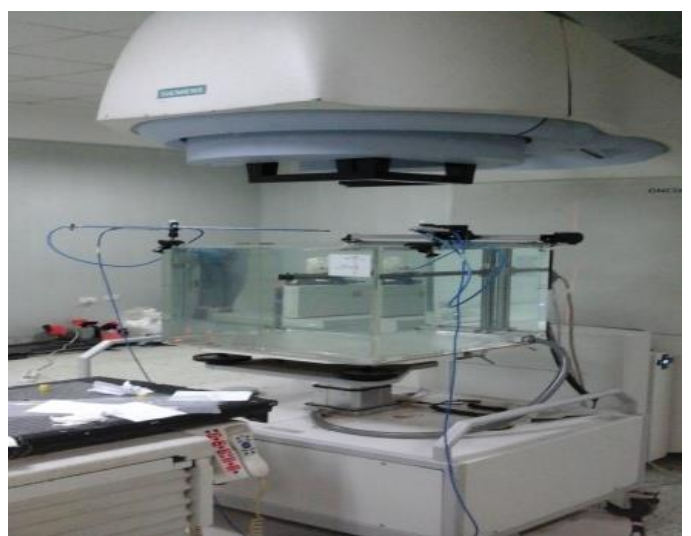

Fig. (2): Water phantom during setting 
A mechanical check has been performed to check the quality of the device for Dosimetric measurements by ensuring that the absorbed dose is constant. Optical laser lines that undermine the cross-wires within the lightweight field were examined. The isocenter for point for gantry, couch rotation and collimator have been checked. After that, the water phantom was changed at 100 $\mathrm{cm}$ SSD and the measuring instrument was found at $10 \mathrm{~cm}$ deep for 6 and $15 \mathrm{MV}$ at a gantry angle of 90 degrees, a zero-degree of collimator angle, and the angle of the couch to zero. This way all conditions are compatible with the International Atomic Energy Agency standards (TRS 398).

The pressure and temperature were measured for calculation of the correction factor that determines the pressure and temperature effect on measurement. Different small fields were irradiated to measure the absorbed dose for each field [9]. The data obtained by TPS were compared with the practical data of the ICs and Array Seven29 in the depth of the maximum dose for each energy value $(1.6 \mathrm{~cm}$ for $6 \mathrm{MV}$ and $2.8 \mathrm{~cm}$ for $15 \mathrm{MV})$. When different fields are irradiated, the absorbed dose is determined for each field, and the results are compared with the TPS data. The pressure and temperature were estimated to calculate the corrective issue for them, which estimates the effect of pressure and temperature on the assay. Various small fields were irradiated entirely to absorb the dose for each field. The TPS was compared with reasonable data from Ionization and Array Seven29 at the depth of most dose per energy $(1.6 \mathrm{~cm}$ to $6 \mathrm{MV}$ and $2.8 \mathrm{~cm}$ to 15 $\mathrm{MV}$ ), once the entire fields irradiated, the absorbed dose for each field was determined, and the results were examined with information TPS.

\section{Results}

Absolute values of dose measurement by ionization chambers

Small field size for $6 \mathrm{MV}$

Table (1) describes the calculated data of the absorbed dose from the XIO treatment coming with the system compared with the measured ionization chamber data, where two types of ionization chambers (Semiflex and Pinpoint) were used for different dimensions at a reference depth of $10 \mathrm{~cm}$ for small field dimensions of $6 \mathrm{MVs}$ Fig (3). The comparison of calculated TPS and standards measured by ionization chambers demonstrated a distinction between calculated and measured data in three regions. The first region represents the distinction between the data calculated by TPS and the data measured by the ionization chamber data of the two small field dimensions $\left(4 \times 4\right.$ and $\left.5 \times 5 \mathrm{~cm}^{2}\right)$ and averaged to 6 $\%$ for ionization chambers used. The second region demonstrated the average distinction to $40 \%$ between the data calculated by TPS and the data measured by the field dimensions $2 \times 2 \mathrm{~cm}^{2}$. The third region showed usual discrepancy between the data calculated by TPS and the data measured by ICs for $1 \times 1 \mathrm{~cm}^{2}$.

Table (1): TPS information compared to the measured values by different Ionization chambers for $6 \mathrm{MV}$

\begin{tabular}{cccc}
\hline Field size & TPS Gy & $\begin{array}{c}\text { Pinpoint } \\
\text { Gy }\end{array}$ & $\begin{array}{c}\text { Semiflex } \\
\text { Gy }\end{array}$ \\
\hline $1 \times 1$ & 0.577 & 0.457 & 0.040 \\
$2 \times 2$ & 0.589 & 0.459 & 0.286 \\
$3 \times 3$ & 0.649 & 0.653 & 0.607 \\
$4 \times 4$ & 0.689 & 0.695 & 0.620 \\
$5 \times 5$ & 0.705 & 0.717 & 0.673 \\
\hline
\end{tabular}

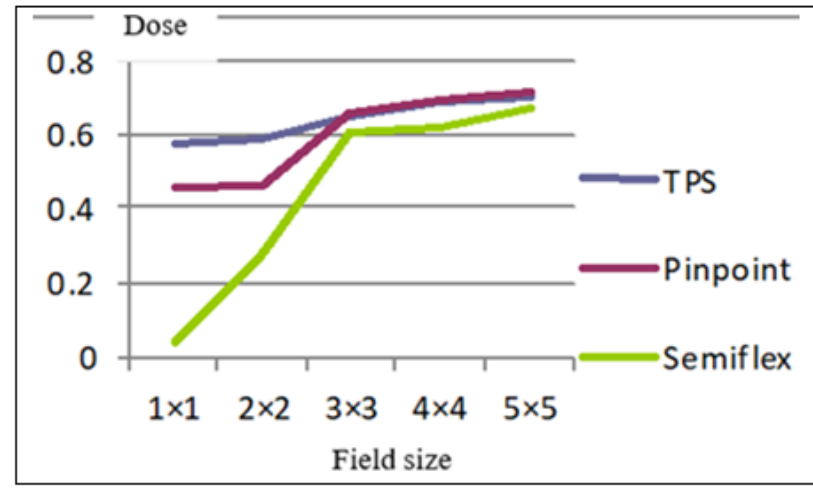

Fig. (3): TPS data versus the measured values by different Ion Chambers for $6 \mathrm{MV}$

\section{Small field size for $15 \mathrm{MV}$}

The absorbed dose was calculated using CMS treatment and compared with the measurements of the ionization chambers Table (2). The two types of ionization chambers (Semiflex and Pinpoint) have been used for different field dimensions at 10 $\mathrm{cm}$ reference depth to field dimensions for $15 \mathrm{MV}$ at a range from $1 \times 1,2 \times 2,3 \times 3,4 \times 4,5 \times 5 \mathrm{~cm}^{2}$ dimensions at $100 \mathrm{~cm}$ SSD Fig. (4). 
Table (2): TPS information comparing with the measured values by different Ionization chambers for $15 \mathrm{MV}$

\begin{tabular}{|c|c|c|c|}
\hline Field size & TPS Gy & $\begin{array}{l}\text { Pinpoint } \\
\text { Gy }\end{array}$ & $\begin{array}{l}\text { Semiflex } \\
\text { Gy }\end{array}$ \\
\hline $1 \times 1$ & 0.587 & 0.458 & 0.040 \\
\hline $2 \times 2$ & 0.588 & 0.460 & 0.288 \\
\hline $3 \times 3$ & 0.659 & 0.653 & 0.606 \\
\hline $4 \times 4$ & 0.690 & 0.696 & 0.621 \\
\hline $5 \times 5$ & 0.705 & 0.718 & 0.672 \\
\hline
\end{tabular}

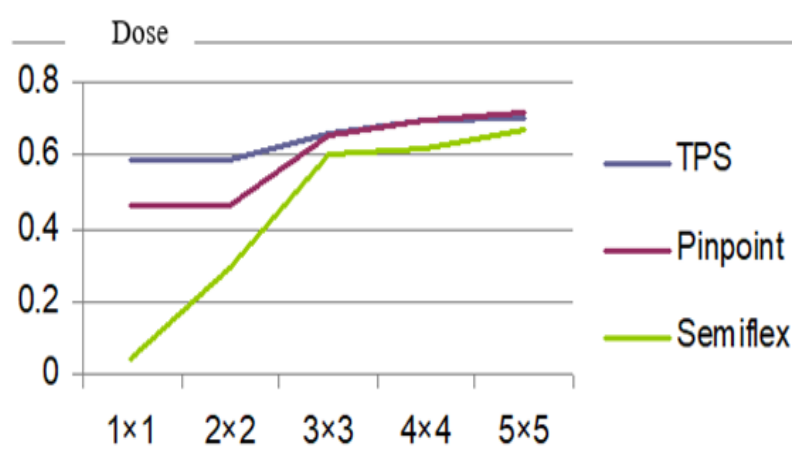

Field size

Fig. (2): TPS data versus the measured values by water phantom using different ion chambers for $15 \mathrm{MV}$

\section{Absolute values of 2D-array dose measurement Small field size for $6 \mathrm{MV}$}

The CMS treatment designing system was used for calculation of absorbed dose information compared to the 2D-Array measured information Table (3) for different small field dimensions (1, 2, 3, 4 and $5 \mathrm{~cm}$ depth for max dose) was $1.6 \mathrm{~cm}$ at $100 \mathrm{MU}$. Table (3) showed slight variations between TPS and the experimental information for fields $2 \times 2$ and $3 \times 3 \mathrm{~cm} 2$ Fig. (5); For $1 \times 1 \mathrm{~cm} 2$ area, the measured information is minimized with in $1.5 \%$. For the $4 \times 4$ $\mathrm{cm} 2$ field, the measured TPS and measured data are the same of $5 \times 5 \mathrm{~cm} 2$ area measures. This is due to the amount of scattered radiation that is directly proportional to the field space.
Table (3): TPS data comparing to the measured values by 2D-Array for $6 \mathrm{MV}$

\begin{tabular}{ccc}
\hline Field size & TPS cGy & 2D array \\
\hline $1 \times 1$ & 86.9 & 84.43 \\
$2 \times 2$ & 89.1 & 87.1 \\
$3 \times 3$ & 90.44 & 88.89 \\
$4 \times 4$ & 91.55 & 91.89 \\
$5 \times 5$ & 93.57 & 92.23 \\
\hline
\end{tabular}

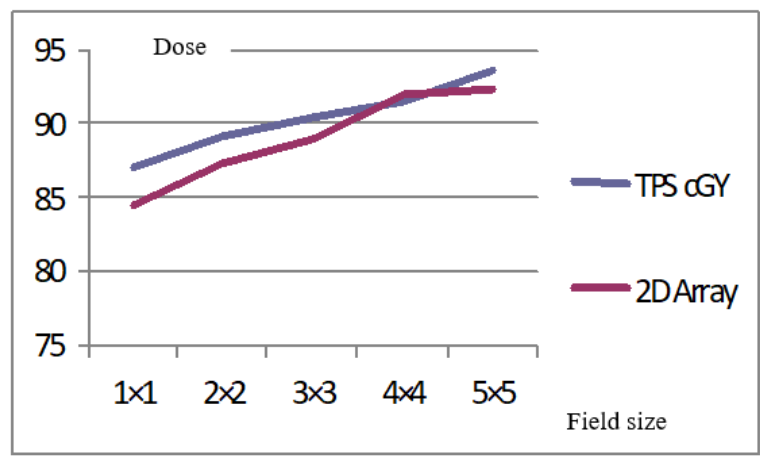

Fig. (5): TPS data versus the measured values by 2DArray for 6 MV

\section{Small field size for $15 \mathrm{MV}$}

The absorbed dose calculation was done by XIO treatment system and compared to the measurements done using two-dimensional array as shown in Table (4), for different small field dimensions (1, 2, 3, 4, and 5) at $100 \mathrm{~cm}$ SSD. Little distinction has been shown between TPS and the measured data in $1 \times 1 \mathrm{~cm}^{2}$ field dimension; 2DArray results were similar in TPS and measured data in one field. The measurements showed an increase when the area was expanded from $2 \times 2$ to $5 \times 5 \mathrm{~cm}^{2}$ according to the fixed constant values $4 \%$. Adjustments have been applied to response deviations due to existing energy as well as response changes due to space and depth. Process variance errors were reduced using a 2D-Array reference standard and processed with each experiment [10]. 


Table (4): TPS data versus the measured results by 2D-
\begin{tabular}{cccc}
\hline \multicolumn{3}{c}{ Array for 15 MV } \\
\hline Field size & TPS cGy & 2D array \\
\hline $1 \times 1$ & 82.43 & 65.27 \\
$2 \times 2$ & 81.32 & 69.53 \\
$3 \times 3$ & 89.25 & 90.56 \\
$4 \times 4$ & 92.21 & 94.22 \\
$5 \times 5$ & 94.01 & 96.71 \\
\hline
\end{tabular}

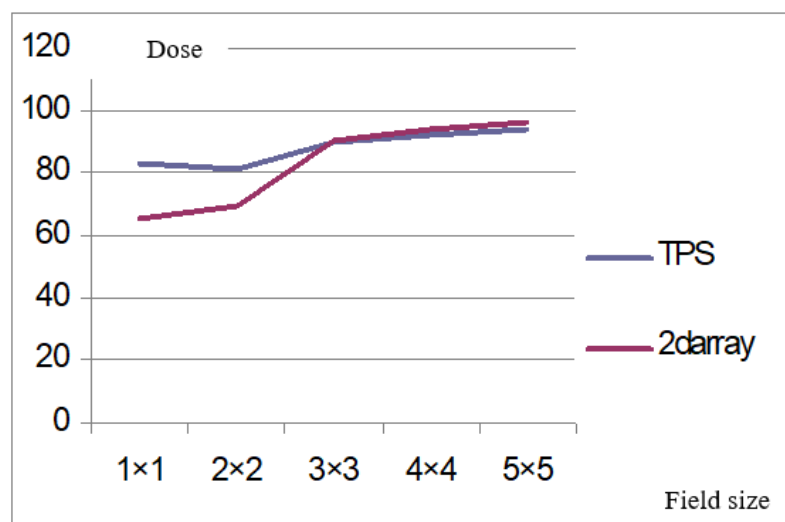

Fig. (6): TPS data versus the measured results by 2DArray for 15 MV

\section{Discussion}

As had shown tin Table (1), The measured doses in the conventional phantom were lower than the TPS values for small field dimensions, smaller than $3 \times 3 \mathrm{~cm}^{2}$; this reduction was due to the nonabundant electron balance of the fully measured scattered of the photon beam accelerator $6 \mathrm{MV}$. Measured doses are in the standard phantom, the TPS values for the_dimensions are smaller than $3 \times 3 \mathrm{~cm}^{2}$. This result can be explained as the electronic balance is uncomfortable for the package of scattered photons from linear accelerator and the photon beam energy was $6 \mathrm{MV}$ and $15 \mathrm{MV}$. On contrary, the field dimensions over $3 \times 3 \mathrm{~cm}^{2}$ showed a slight difference between TPS value and the experimental data for fields $1 \times 1,2 \times 2$ and $3 \times 3 \mathrm{~cm}^{2}$. A remarkable difference at intervals pair of data, experimental data for field $4 \times 4 \mathrm{~cm}^{2}$ and $5 \times 5 \mathrm{~cm}^{2}$ was observed and increased with pair of information accumulated at intervals five-hitter. These results could be due to the scattered radiation amount that is directly proportionate with field space information.

Table (2) shows that the inconsistencies between calculated information and measured values are confirmed in a two- region unit. The first one represents distinction between the calculated information by TPS and measured information by ion chambers ICs for small field dimensions $(4 \times 4$ and $5 \times 5 \mathrm{~cm}^{2}$ ) averaged to $4 \%$ for the particle chambers utilized in gift work. This information was in line with information revealed by Vieillevigne et al. (2018) [8], $\left(4 \times 4\right.$ and $\left.5 \times 5 \mathrm{~cm}^{2}\right)$, with an average of $4 \%$ for ion chamber used in gift making. This information conforms to the information disclosed [9], with a $3.8 \%$ link contrast rate. The second area shows a typical discrepancy of $20 \%$ between the TPS and the information measured by the ion chamber of the dimensions $\left(1 \times 1\right.$ and $\left.2 \times 2 \mathrm{~cm}^{2}\right)$. The measured doses in the common phantom, TPS values for small field dimensions are smaller than $3 \times 3 \mathrm{~cm}^{2}$. The_low rates were believed to be due to insufficient electronic balance for scattered photons from linear accelerator photon beam energies 6 and 15MV.

In Table (2), TPS information was compared with the measured values by different Ionization chambers for $15 \mathrm{MV}$ confirmed in the two- region unit, as shown below. The first region represents distinction between the calculated information by TPS and measured information by ion chambers ICs for small field dimensions, $\left(4 \times 4\right.$ and $\left.5 \times 5 \mathrm{~cm}^{2}\right)$ averaged to $4 \%$ for the particle chambers utilized in gift work. This information was in line with information revealed by Vieillevigne et al. (2018) [8], $\left(4 \times 4\right.$ and $\left.5 \times 5 \mathrm{~cm}^{2}\right)$, with average to $4 \%$ for ion chamber used in gift making. This information conforms to the information disclosed [9] with a $3.8 \%$ link contrast rate. The second area shows the typical discrepancy of $20 \%$ between the TPS and the information measured by the ionchamber of the dimensions $\left(1 \times 1\right.$ and $\left.2 \times 2 \mathrm{~cm}^{2}\right)$. The measured doses in the common phantom, TPS values for small field dimensions are smaller than $3 \times 3 \mathrm{~cm}^{2}$, The low rates were believed to be due to insufficient electronic balance for scattered photons from linear accelerator photon beam energies 6 and 15MV.

Little differences were shown between TPS and the experimental data for fields $2 \times 2$ and $3 \times 3 \mathrm{~cm}^{2}$ Table (3). These differences are due to $\pm 2 \%$ intervals of the smart resolution of the menstrual design planning system. For $1 \times 1 \mathrm{~cm}^{2}$ field size, the measured information is minimized with in $1.5 \%$. For the $4 \times 4 \mathrm{~cm}^{2}$ field, the measured TPS and measured data are the same of $5 \times 5 \mathrm{~cm}^{2}$ field measurements. This is due to the amount of 
scattered radiation that is directly proportional to the field space [11].

\section{Conclusion}

Water phantom measured doses were estimated and TPS values for small field dimensions; for fields smaller than $3 \times 3 \mathrm{~cm}^{2}$. The low rates were believed to be due to insufficient electronic balance for scattered photons from linear accelerator photon beam energies 6 and 15MV. On the other hand, field dimensions greater than $3 \times 3 \mathrm{~cm}^{2}$ have a mean of $7 \%$ for Semiflex, Pinpoint and Farmer ionized chamber utilized in this study to $6 \mathrm{MV}$ whereas $15 \mathrm{MV}$ revealed 3\% average for all used ionized chambers. The data measured using 2D-Array for $6 \mathrm{MV}$ illustrated little variations between the TPS values and so the experimental data for fields $2 \times 2$ and $3 \times 3 \mathrm{~cm}^{2}$ were within the acceptable value of $\pm 2 \%$ is due to the accuracy of the activity designing system. In case of field size of $1 \times 1 \mathrm{~cm}^{2}$, the measured data was reduced within $1.5 \%$ and for field $4 \times 4 \mathrm{~cm}^{2}$, the measured data increased within $6 \%$, due to the quantity of scattered amount of radiation which is proportionately directed to field size. A gentile difference was found between the TPS values and the experimental data imposition 2D-Array for $15 \mathrm{MV}$. The results were equal for TPS values and measured data at $1 \times 1 \mathrm{~cm}^{2}$ field dimension. The measured data could be increased by increasing of field space from $2 \times 2$ to $5 \times 5 \mathrm{~cm}^{2}$ using $4 \%$ constant worth.

\section{References}

1-Dewaraja, Y. K., Frey, E. C., Sgouros, G., Brill, A. B., Roberson, P., Zanzonico, P. B., \& Ljungberg, M. (2012) MIRD pamphlet no. 23: quantitative SPECT for patient-specific 3-dimensional dosimetry in internal radionuclide therapy. Journal of Nuclear Medicine, 53(8), 1310-1325.

2-Lutz, S., Berk, L., Chang, E., Chow, E., Hahn, C., Hoskin, P., ... \& Sahgal, A. (2011) Palliative radiotherapy for bone metastases: an ASTRO evidence-based guideline. International Journal of Radiation Oncology* Biology* Physics, 79(4), 965976.

3-Das, I. J., Cheng, C. W., Watts, R. J., Ahnesjö, A., Gibbons, J., Li, X. A., ... \& Zhu, T. C. (2013) Accelerator beam data commissioning equipment and procedures: report of the TG-106 of the therapy physics commit

4-ee of the AAPM. Igaku butsuri: Nihon Igaku Butsuri Gakkai kikanshi= Japanese journal of medical physics: an official journal of Japan Society of Medical Physics, 33(1), 16.

5-Li, J., Wiersma, R. D., Stepaniak, C. J., Farrey, K. J., \& Al-Hallaq, H. A. (2012) Improvements in dose accuracy delivered with static-MLC IMRT on an integrated linear accelerator control system. Medical physics, 39(5), 2456-2462.

6-Scott, A. J., Kumar, S., Nahum, A. E., \& Fenwick, J. D. (2012) Characterizing the influence of detector density on dosimeter response in non-equilibrium small photon fields. Physics in Medicine \& Biology, 57(14), 4461.

7-Chen, W., Unkelbach, J., Trofimov, A., Madden, T., Kooy, H., Bortfeld, T., \& Craft, D. (2012) Including robustness in multi-criteria optimization for intensitymodulated proton therapy. Physics in Medicine \& Biology, 57(3), 591..

8-Thoelking, J., Sekar, Y., Fleckenstein, J., Lohr, F., Wenz, F., \& Wertz, H. (2016) Characterization of a new transmission detector for patient individualized online plan verification and its influence on 6MV Xray beam characteristics. Zeitschrift für Medizinische Physik, 26(3), 200-208.

9-Vieillevigne, L., \& Arnaud, F. X. (2018) Dosimetric performance of the new PTW 31022 PinPoint 3D ionization chamber in high-energy photon beams. Biomedical Physics \& Engineering Express, 4(4), 047002..

10-Cruz, W., Narayanasamy, G., Papanikolaou, N., \& Stathakis, S. (2015) Dosimetric comparison of water phantoms, ion chambers, and data acquisition modes for LINAC characterization. Radiation Measurements, 82, 108-114.

11-Huq, M. S., Hwang, M. S., Teo, T. P., Jang, S. Y., Heron, D. E., \& Lalonde, R. J. (2018) A dosimetric evaluation of the IAEA-AAPM TRS 483 code of practice for dosimetry of small static fields used in conventional linac beams and comparison with IAEA TRS-398, AAPM TG 51, and TG 51 Addendum protocols. Medical physics, 45(9), 4257-4273.

12-Keivan, H., Shahbazi-Gahrouei, D., Shanei, A., \& Amouheidari, A. (2018) Assessment of Imprecise Small Photon Beam Modeling by Two Treatment Planning System Algorithms. Journal of medical signals and sensors, 8(1), 39. 